\title{
Estimation of Sinusoidally Modulated Signal Parameters Based on the Inverse Radon Transform
}

\author{
Miloš Daković, Ljubiša Stanković \\ Faculty of Electrical Engineering, University of Montenegro, Podgorica, Montenegro \\ E-mail: milos@ac.me, ljubisa@ac.me
}

\begin{abstract}
A method for accurate and efficient parameters estimation and decomposition of sinusoidally modulated signals is presented. This kind of signals is of special interest in radars and communications. The proposed method is based on the inverse Radon transform property to transform a twodimensional sinusoidal pattern into a single point in a twodimensional plane. Theory is illustrated on signals with one and more components, including noise and disturbances, as well as time-frequency patterns that deviate from sinusoidal form, including the cases when some samples are not available.
\end{abstract}

Keywords- Frequency modulation, Parameter estimation, Radon transform, Time-frequency signal analysis

\section{INTRODUCTION}

Sinusoidally modulated signals appear in many applications, like in radars and communications. In radar signal processing fast rotating, vibrating or oscillating parts reflect a signal causing micro-Doppler effect in a form of sinusoidally modulated signal. In practice it is very important to extract, decompose, and estimate parameters of this kind of signals, since they are easily related to the physical dimensions and other properties of moving objects. Most of the techniques used for the detection, extraction and parameters estimation of these signals are based on two approaches. One is the parametric approach when the form of signal, we are looking for, is assumed and we try to extract a desired component by matching its parameters [1],[2]. Other approach is based on the L-statistics and time-frequency analysis to extract non-stationary features from the time-frequency representation of a composite signal. This method just separate stationary and non-stationary parts, but it does not separate non-stationary components within the signal [3],[4]. Here we will present a method for analysis of sinusoidally modulated components based on the inverse Radon transform of signal's time-frequency representation. The Radon transform, widely used in computer imaging applications, is also used in time-frequency for projecting Wigner distribution in order to detect linear frequency modulated signals [5]-[9]. Here, we will use the inverse Radon transform rather than the Radon transform. Note, that the behavior of the direct and inverse Radon transform is completely different, in contrast, for example, to the Fourier transform [10]. Since the Radon transform of a two dimensional signal containing a two-dimensional delta function is a sinusoidal pattern with amplitude corresponding to the distance of the point from the origin and the initial phase corresponding to the phase of the point position, then it is obvious that a sinusoidal pattern in the time-frequency plane (produced by a time-frequency representation of sinusoidally modulated signal) will project to a two-dimensional delta in the inverse Radon transform.
This is obviously an optimal transform for a two-dimensional sinusoidal pattern, since all signal energy from the timefrequency domain will be projected in a single point in inverse Radon transform domain. The method will be introduced on monocomponent sinusoidally modulated signal. Then it will be extended to noisy and multicomponent signals that include one or more sinusoidal patterns. Finally the method will be applied to periodic and non-periodic patterns that are not produced by a sinusoidally modulated signals at all. The examples illustrate the efficiency of the presented method.

The paper is organized as follows. The inverse Radon transform is reviewed in Section II. A method for estimation of the sinusoidally modulated signal parameters is the topic of Section III.

\section{RADON AND INVERSE RADON TRANSFORM}

A projection of a two-dimensional function $f(x, y)$ onto the $x$-axis is

$$
R_{f}(x)=\int_{-\infty}^{\infty} f(x, y) d y
$$

A rotated version of a two-dimensional signal may be described in a rotated coordinate system, by a coordinate rotation transform. For an angle $\alpha$, it reads

$$
\left[\begin{array}{l}
\xi \\
\zeta
\end{array}\right]=\left[\begin{array}{rr}
\cos (\alpha) & \sin (\alpha) \\
-\sin (\alpha) & \cos (\alpha)
\end{array}\right]\left[\begin{array}{l}
x \\
y
\end{array}\right]
$$

The projection of a function $f(x, y)$ onto $\xi$, with a varying rotation angle $\alpha$, is the Radon transform of the signal $f(x, y)$

$$
R_{f}(\xi, \alpha)=\int_{-\infty}^{\infty} f(\xi, \zeta) d \zeta=\int_{-\infty}^{\infty} \int_{-\infty}^{\infty} f(\lambda, \zeta) \delta(\lambda-\xi) d \lambda d \zeta .
$$

Let us consider simple setup where the analyzed image is twodimensional delta function located at the point $f(x, y)=\delta(x-$ $\left.x_{0}\right) \delta\left(y-y_{0}\right)$ in $x, y$ domain. Projection of $f(x, y)$ onto $x$ axis is

$$
R_{f}(x, 0)=\int_{-\infty}^{\infty} f(x, y) d y=\delta\left(x-x_{0}\right)
$$

For an arbitrary direction $\xi_{0}=x_{0} \cos (\alpha)+y_{0} \sin (\alpha), \zeta_{0}=$ $-x_{0} \sin (\alpha)+y_{0} \cos (\alpha)$, the function $f(\xi, \zeta)=\delta\left(\xi-\xi_{0}\right) \delta(\zeta-$ 
$\left.\zeta_{0}\right)$ results in the Radon transform

$$
\begin{aligned}
R_{f}(\xi, \alpha) & =\int_{-\infty}^{\infty} f(\xi, \zeta) d \zeta=\delta\left(\xi-\xi_{0}\right) \\
& =\delta\left(\xi-\left(x_{0} \cos (\alpha)+y_{0} \sin (\alpha)\right)\right) .
\end{aligned}
$$

Note that this is a sinusoidal pattern in a two-dimensional $(\xi, \alpha)$ domain, with the amplitude $\sqrt{x_{0}^{2}+y_{0}^{2}}$ and the phase $\psi=\arctan \left(y_{0} / x_{0}\right)$. Of course, the Radon transform is periodic in $\alpha$ with $2 \pi$. Projections for $0 \leq \alpha<\pi$ are sufficient to calculate all transform values. By knowing all the projections, for $0 \leq \alpha<\pi$, we can calculate the two-dimensional Fourier transform of $f(x, y)$. It means that we can reconstruct a twodimensional function $f(x, y)$ from its projections or integrals (basic theorem for computed tomography). The inverse Radon transform may be calculated in the Fourier domain or by projecting back the Radon transform (back-projection method). Thus, a point in the $(x, y)$ domain transforms to a sinusoidal pattern in Radon transform domain. It means that a sinusoidal pattern will be transformed into a point by using the inverse Radon transform (IRT). When all energy is concentrated into a point, then its parameters estimation is very robust and reliable.

\section{PARAMETERS ESTIMATION}

Let us now consider sinusoidally frequency modulated signal

$$
x(t)=A_{x} \exp \left(j \frac{A_{m}}{f_{m}} \sin \left(2 \pi f_{m} t+\theta_{m}\right)\right) .
$$

It is known that a time-frequency representation $T(t, \omega)$ of a given signal concentrate the signal energy along the signal instantaneous frequency

$$
\omega_{i}(t)=2 \pi A_{m} \cos \left(2 \pi f_{m} t+\theta_{m}\right)
$$

i.e. this signal in $(t, \omega)$ plane is presented as sinusoidal pattern image (with more or less deviations depending on the timefrequency representation used to transform the signal into timefrequency domain). If we change time coordinate with $\varphi=$ $2 \pi f_{m} t$ then, from the previous section we can conclude that the IRT of the obtained image $T\left(\varphi /\left(2 \pi f_{m}\right), \omega\right)$ reduces to single point where distance form origin correspond to modulation parameter $A_{m}$ and the angle of the point is equal to $\theta_{m}$. In this way we can accurately estimate modulation parameters $A_{m}$ and $\theta_{m}$.

Modulation parameter $f_{m}$ can be estimated in the following way. Let us introduce coordinate change from $t$ to $\varphi$ as $\varphi=\alpha t$ where $\alpha$ is parameter. Now we can vary parameter $\alpha$ within some range of possible values and search for the value $\hat{\alpha}$ that produces single point IRT. In that case we know that $\hat{\alpha}=$ $2 \pi f_{m}$ and we can estimate modulation parameter $f_{m}$. In this procedure it is needed to find the value of $\alpha$ when the IRT reduces to a single point within the considered domain. This mean that the IRT is ideally concentrated and the concentration measures [7] can be used to detect that we reached $\hat{\alpha}$. Range for $\alpha$ should be wide enough to include $2 \pi f_{m}$. Its limits could be determined as the minimal and the maximal expected $2 \pi f_{m}$ in the considered case.

The estimation algorithm is summarized as:
Step 1. Start from a frequency modulated signal $x(t)$ with unknown modulation parameters. Assume that modulation frequency satisfies $f_{\min } \leq f_{m} \leq f_{\max }$, where $f_{\min }$ and $f_{\max }$ are constants.

Step 2. Calculate time-frequency representation $T(t, \omega)$ of $x(t)$. Here we can use any time-frequency representation [11] concentrating the signal energy along the instantaneous frequency in the time-frequency plane. The result of this step is a two-dimensional time-frequency image of the considered signal.

Step 3. Consider a set of possible $\alpha$ as $M$ equally spaced values between $2 \pi f_{\min }$ and $2 \pi f_{\max }$.

Step 4. For each $a$ within considered set introduce coordinate change $\varphi=\alpha t$ and calculate the IRT of the image $T(\varphi / \alpha, \omega)$.

Step 5. Calculate the concentration measure $\mu$ of the obtained IRT for each $\alpha$ and find $\hat{\alpha}$ that provide the highest concentration.

Step 6. Estimate modulation frequency as $\hat{f}_{m}=\hat{\alpha} /(2 \pi)$.

Step 7. Find position of IRT maximum calculated with $\hat{\alpha}$, i.e. IRT of $T(\varphi / \hat{\alpha}, \omega)$. Denote the detected coordinates as $x_{m}$ and $y_{m}$.

Step 8. Estimate the modulation amplitude as

$$
\hat{A}_{m}=\sqrt{x_{m}^{2}+y_{m}^{2}} .
$$

Step 9. Estimate the modulation phase as $\hat{\theta}_{m}=\arctan \frac{y_{m}}{x_{m}}$.

In the case of non-sinusoidally modulated signals, producing non-sinusoidal patterns in the time-frequency plane, the presented approach will produce the closest sinusoidal pattern form, as it will be shown in examples.

\section{A. Method Implementation}

We use the spectrogram and the S-method as timefrequency representations in the algorithm Step 2. The spectrogram is defined as a squared modulus of the short-time Fourier transform. In the discrete time domain it reads

$$
\begin{aligned}
& \operatorname{SPEC}(n, k)=|\operatorname{STFT}(n, k)|^{2} \\
& \operatorname{STFT}(n, k)=\sum_{m=0}^{N_{w}} w(m) x(n+m) e^{-j \frac{2 \pi}{N_{w}} m k},
\end{aligned}
$$

where $w(n)$ is the analysis window of the length $N_{w}$. Along with the spectrogram, we will use the S-method as a time frequency representation. The discrete S-method is of the form

$$
\begin{aligned}
S M(n, k) & =|\operatorname{STFT}(n, k)|^{2} \\
& +2 \operatorname{Re}\left[\sum_{p=1}^{L} \operatorname{STFT}(n, k+p) \operatorname{STFT}^{*}(n, k-p)\right],
\end{aligned}
$$

where beside the time-domain window, used in the STFT calculation, we have a parameter $L$ that corresponds to the number of spectrogram correcting terms [11]. It is known that the S-method can produce highly concentrated time-frequency representation of a given signal. The S-method is numerically very efficient since there is no need for signal oversampling. 
Two special cases of the S-method are the spectrogram (with $L=0$ ) and the pseudo Wigner distribution (with $L=N_{w} / 2$ ).

The concentration measure is needed in the algorithm, Step 5. Here we use the normalized measure

$$
\mu=\frac{\mathcal{M}_{1}^{1}}{\mathcal{M}_{1 / 2}^{1 / 2}}
$$

where $\mathcal{M}_{p}^{p}$ is defined in [7] as

$$
\mathcal{M}_{p}^{p}=\left(\sum_{n} \sum_{k}|T(n, k)|^{1 / p}\right)^{p}
$$

and $T(n, k)$ are the discrete samples of a non-negative part of the IRT.

Example 1: Consider $N=129$ samples of noise free FM signal (4) sampled with $t=n \Delta t, \Delta t=1 / 128$, $n=0,1, \ldots N-1$. The signal parameters are $A_{x}=1$, $f_{m}=1.4, A_{m}=50$ and $\theta_{m}=30^{\circ}$ The spectrogram and the S-method of the considered signal are presented in Fig. 1(a) and (b). The spectrogram is calculated with a 17point Hann window, while the S-method is calculated with a 55 point Hann window and $L=8$. In both cases the timefrequency representation is calculated at each available time instant (i.e., with overlapping). Parameter $\alpha$ is varied from 0.2 to 15 with step 0.2 . For each $\alpha$ the IRT, along with the corresponding concentration measure, is calculated. The concentration measure $\mu(\alpha)$ is presented in Fig. 1(c) and (d), where the minimum of this measure (i.e. highest concentration) is depicted by a circle.

Fig. 1(e) and (f) presents the IRT obtained for $\hat{\alpha}=8.8$. Maximum position in the IRT is determined and the modulation parameters are estimated in the spectrogram case as

$$
\hat{f}_{m}=\frac{8.8}{2 \pi} \approx 1.4, \quad \hat{A}_{m}=49.1, \quad \hat{\theta}_{m}=31.4^{\circ}
$$

and in the S-method case as

$$
\hat{f}_{m}=\frac{8.8}{2 \pi} \approx 1.4, \quad \hat{A}_{m}=48.1, \quad \hat{\theta}_{m}=32.2^{\circ}
$$

As we can see in both cases modulation parameters are very close to true values. It means that the method will not be too sensitive with respect to the time-frequency representation.

The IRT for $\alpha=7$ (in the spectrogram case) and $\alpha=10$ (in the S-method case) are given in Fig. 1(g) and (h). These subplots illustrate that the IRT for optimal $\alpha$ (subplots (c) and (d)) is better concentrated than the IRT with another $\alpha$.

Example 2: The estimation procedure is applied to a noisy signal $s(n \Delta t)=x(n \Delta t)+\varepsilon(n)$, where the noise $\varepsilon(n)$ is a complex white Gaussian noise with $S N R=0 \mathrm{~dB}$. The results are presented in Fig. 2(a) and (b). The spectrogram of $s(n)$ is presented in Fig. 2(a), while the concentration measure of the IRT, for various $\alpha$, is given in Fig. 2(b). In the spectrogram calculation the number of frequency points is 101, i.e. windowed signal is zero-padded prior to the DFT calculation. Based on the IRT obtained for optimal $\alpha$ (denoted by circle in Fig. 2(b)) the modulation parameters are estimated as

$$
\hat{f}_{m}=\frac{8.8}{2 \pi} \approx 1.4, \quad \hat{A}_{m}=49.1, \quad \hat{\theta}_{m}=30.2^{\circ}
$$
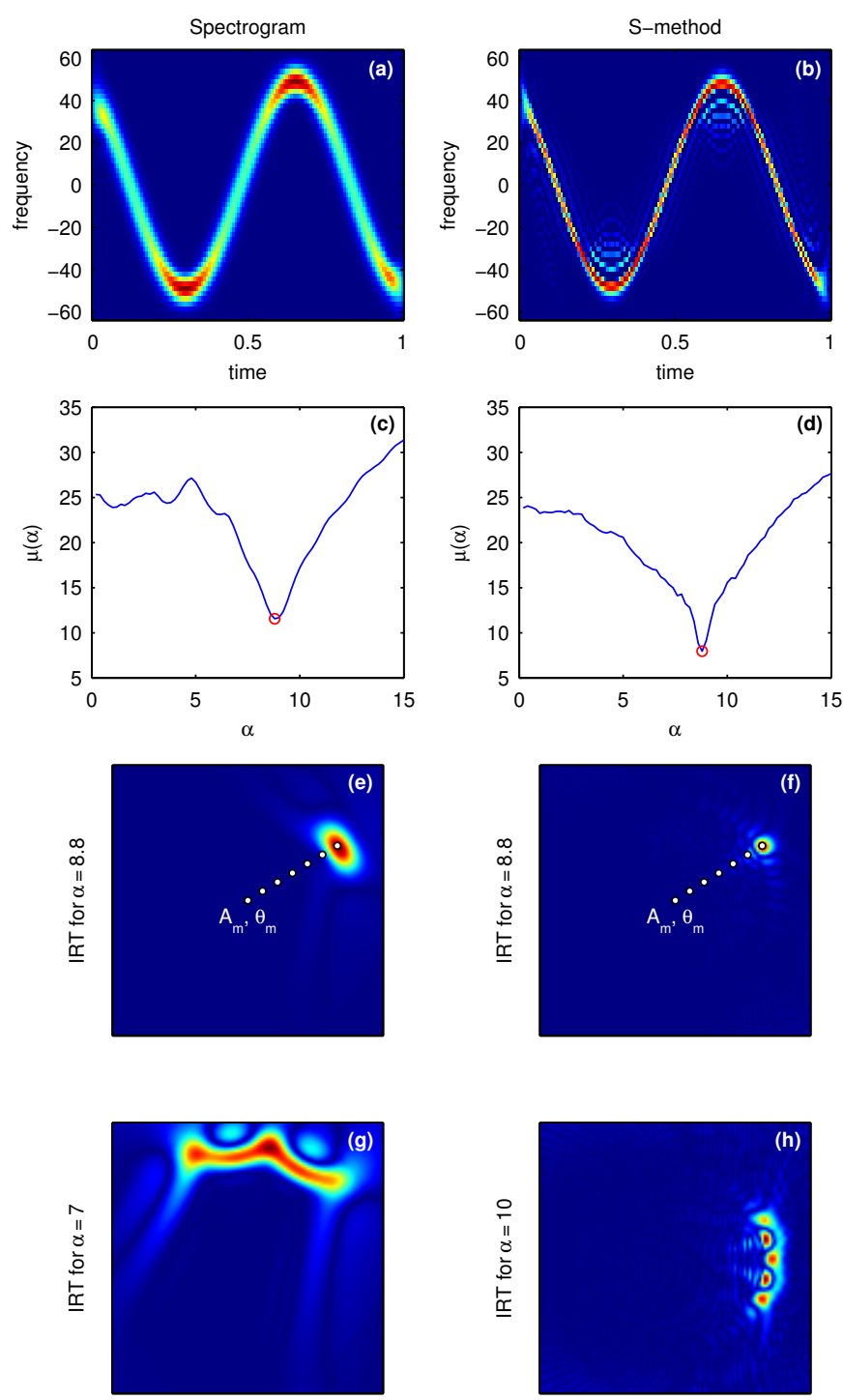

Fig. 1. Modulation parameters estimation for mono-component non-noisy sinusoidally FM signal. Time frequency representation (a), (b); concentration measure (c), (d); inverse Radon transform with highest concentration (e), (f) and inverse radon transform when parameter $\alpha$ is not optimally chosen $(\mathrm{g})$, (h).

and the resulting sinusoidal modulation is plotted over the spectrogram with a black line Fig. 2(a). The estimated parameters are very close to the parameters estimated for noisy free case.

A multicomponent signal composed from a sinusoidally FM component (the same as in Example 1), a linear FM component, and a constant frequency component,

$$
s(t)=x(t)+0.6 \exp \left(j 40 \pi(t-0.8)^{2}\right)+0.6 \exp (j 50 \pi t)
$$

is considered. The results obtained with the proposed procedure are presented in Fig. 2(c) and (d). Estimated parameters are

$$
\hat{f}_{m}=\frac{8.8}{2 \pi} \approx 1.4, \quad \hat{A}_{m}=49.1, \quad \hat{\theta}_{m}=30.2^{\circ} .
$$



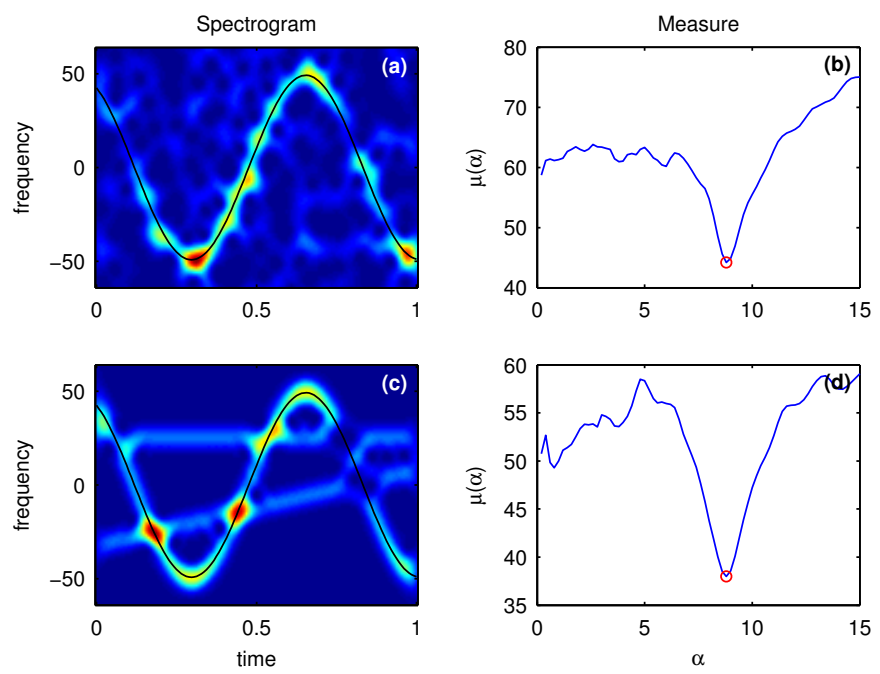

Fig. 2. Parameter estimation of noisy signal with $\mathrm{SNR}=0 \mathrm{~dB}(\mathrm{a})$, (b) and multicomponent signal (c), (d). Estimated modulation is plotted with black line over spectrogram image.

From this example we see that the proposed method is robust to the noise and some other possible interferences.

\section{B. Multicomponent Signal Analysis}

This approach may be generalized to a multicomponent signal

$$
x(t)=\sum_{k=1}^{K} A_{x}^{(k)} \exp \left(j \frac{A_{m}^{(k)}}{f_{m}^{(k)}} \sin \left(2 \pi f_{m}^{(k)} t+\theta_{m}^{(k)}\right)\right)+\varepsilon(t),
$$

where $\varepsilon(t)$ denotes disturbing components and noise. Two scenarios are possible. One is that in the application of the previous algorithm, in Step 5, the concentration measure $\mu$ of the obtained IRT produces at once several or all $K$ values of $\alpha$ with visible and distinguishable concentration measure peaks. Then, these values are associated to the corresponding signal parameters, as in Steps 6-9. However, due to different amplitudes and different number of periods in the timefrequency plane usually only the strongest component is visible in the concentration measure. In this case its parameter $\hat{\alpha}$ is estimated as in Step 5. The other parameters are estimated for this component as in Steps 6-9. The strongest component is removed and the algorithm is used on the remaining signal components, until the energy of the remaining signal is negligible. After parameters of all components are found, they can be readjusted by a mean-squared comparison with the original signal.

Example 3: Let us consider a multicomponent noisy signal consisted of $K=3$ sinusoidally FM components of the form (5). Signal parameters are:

$$
\begin{aligned}
& A_{x}^{(1)}=1, f_{m}^{(1)}=1.4, A_{m}^{(1)}=50, \theta_{m}^{(1)}=30^{\circ}, \\
& A_{x}^{(2)}=0.7, f_{m}^{(2)}=1, A_{m}^{(2)}=35.7, \theta_{m}^{(2)}=-60^{\circ}, \\
& A_{x}^{(3)}=0.7, f_{m}^{(3)}=0.8, A_{m}^{(3)}=28.6 \text { and } \theta_{m}^{(3)}=180^{\circ} .
\end{aligned}
$$
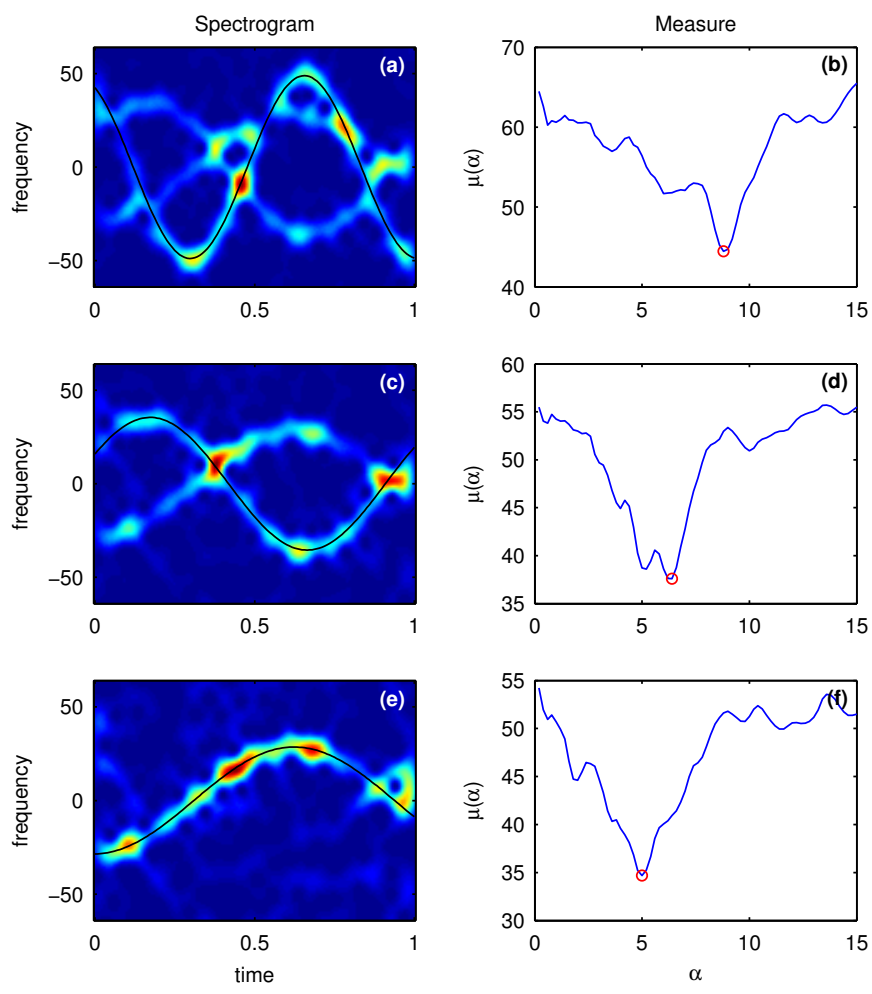

Fig. 3. Multicomponent signal. Estimation of the first component (a), (b); second component (c), (d); and third component (e) and (f). Each component is removed from the signal after estimation, according to the described procedure, prior to next component estimation.

The proposed method estimate parameters of one component, as presented in Fig. 3(a) and (b). The estimated parameters are

$$
\hat{f}_{m}^{(1)}=\frac{8.8}{2 \pi} \approx 1.4, \quad \hat{A}_{m}^{(1)}=48.8, \quad \hat{\theta}_{m}^{(1)}=29.6^{\circ}
$$

From Fig. 3(a) we can see that estimated modulation parameters highly correspond to the component instantaneous frequency. Now we will filter out the estimated component.

In the filtering procedure the original signal is demodulated

$$
x_{d}(n)=x(n) \exp \left(-j \frac{\hat{A}_{m}}{\hat{f}_{m}} \sin \left(2 \pi \hat{f}_{m} n \Delta t+\hat{\theta}_{m}\right)\right) .
$$

The DFT of the demodulated signal $X_{d}(k)=\operatorname{DFT}\left[x_{d}(n)\right]$ is calculated and DC component is removed by putting zero value to $X_{d}(0)$, and several neighboring points $X_{d}(1), X_{d}(N)$, $X_{d}(2), X_{d}(N-1) \ldots$ Here the signal length is $N=129$ and we remove 7 points. The filtered signal is obtained by the inverse DFT $x_{f}(n)=\operatorname{IDFT}\left[X_{d}(k)\right]$.

Finally filtered signal is modulated in order to cancel frequency shifts in the remaining components caused by the demodulation

$$
x_{m}(n)=x_{f}(n) \exp \left(j \frac{\hat{A}_{m}}{\hat{f}_{m}} \sin \left(2 \pi \hat{f}_{m} n \Delta t+\hat{\theta}_{m}\right)\right) .
$$



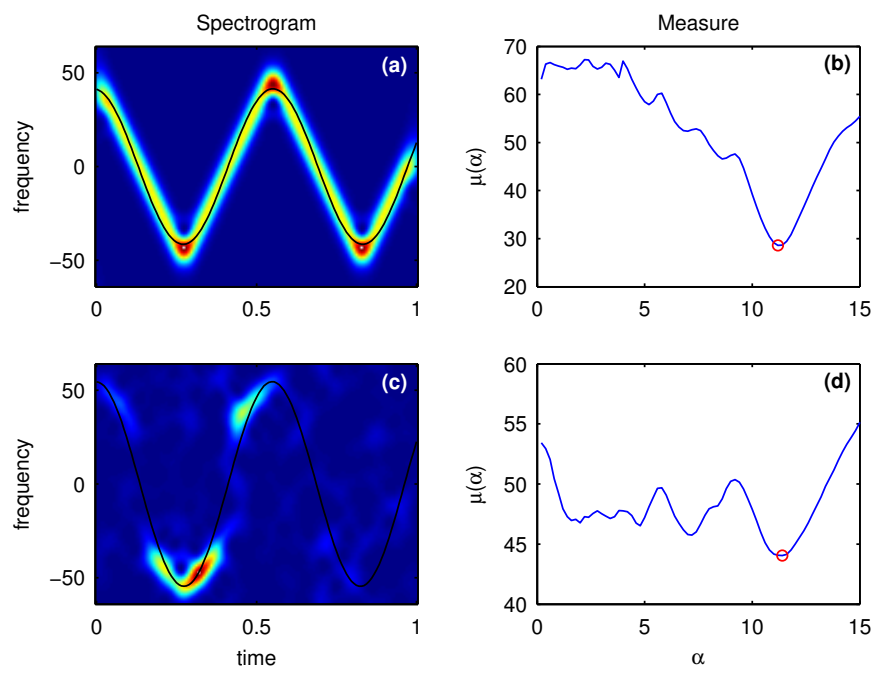

Fig. 4. Nonsinusoidal modulation. Triangularly modulated signal (a), (b); Signal with nonsinusoidal modulation and varying amplitude (c), (d).

Now we can repeat estimation procedure with $x(n)=$ $x_{m}(n)$ and estimate second component parameters. The results are presented in Fig. 3(c) and (d). The estimated modulation parameters are

$$
\hat{f}_{m}^{(2)}=\frac{6.4}{2 \pi} \approx 1.02, \quad \hat{A}_{m}^{(2)}=35.4, \quad \hat{\theta}_{m}^{(2)}=-63.4^{\circ}
$$

In the next step we filter the estimated component and proceed to the parameters estimation for the last component. Results are given in Fig. 3(e) and (f) and the estimated parameters are

$$
\hat{f}_{m}^{(3)}=\frac{5}{2 \pi} \approx 0.796, \quad \hat{A}_{m}^{(3)}=28.5, \quad \hat{\theta}_{m}^{(3)}=-178.7^{\circ} .
$$

The agreement with the true parameters is high.

\section{Nonsinusoidally Modulated Signals}

The presented estimation procedure could be used even if the analyzed signal is periodic, but not sinusoidally modulated. We will illustrate this application on an example.

Example 4: Considered a triangularly modulated signal $x_{1}(t)$ and nonsinusoidal periodic modulated signal $x_{2}(t)$ with a varying amplitude

$$
\begin{aligned}
& x_{1}(t)=\exp \left(j \int_{0}^{t} 200 \arcsin (\cos (3.6 \pi u)) d u\right) \\
& x_{2}(t)=A(t) \exp \left(j \int_{0}^{t} 300 \sqrt[3]{\arcsin (\cos (3.6 \pi u))} d u\right)
\end{aligned}
$$

where

$$
A(t)=\exp \left(-\left(\frac{t-0.35}{0.3}\right)^{2}\right)
$$

Although the proposed method is derived having in mind sinusoidal modulation, the results presented in Fig. 4 clearly show that the applicability of the proposed method is not limited to the sinusoidal modulation patterns only. The estimated modulation parameters for signal $x_{1}(t)$ are

$$
\hat{f}_{m}=\frac{11.2}{2 \pi} \approx 1.78, \quad \hat{A}_{m}=41.4, \quad \hat{\theta}_{m}=6.1^{\circ}
$$
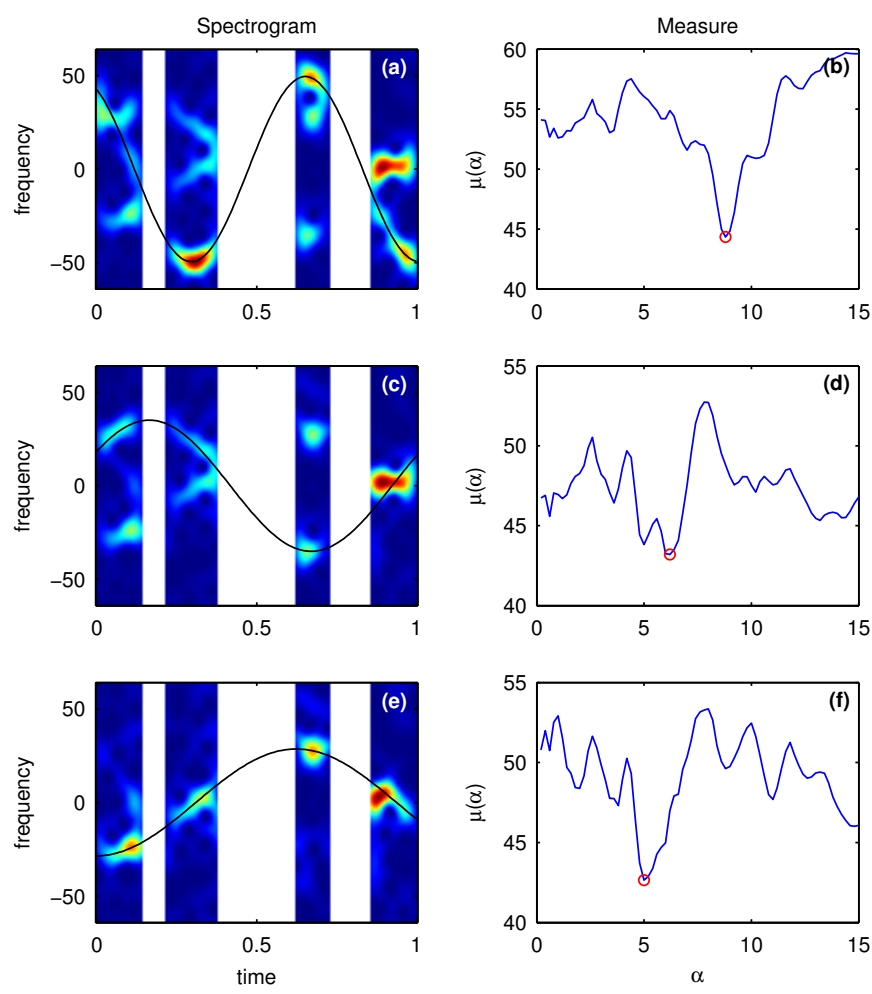

Fig. 5. Multicomponent signal with missing intervals. First component estimation (a), (b); Second component (c), (d); Third component (e), (f). Missing values in time-frequency representations (a), (c) and (e) are presented in white color.

and for signal $x_{2}(t)$

$$
\hat{f}_{m}=\frac{11.4}{2 \pi} \approx 1.81, \quad \hat{A}_{m}=54.5, \quad \hat{\theta}_{m}=1.33^{\circ} .
$$

They agree with $f_{m}=1.8$ in the considered signals. The closest estimated sinusoids are presented in this figure as well.

\section{Analysis Using Partial Data}

Assume that not all signal samples are available. In this case we can calculate the spectrogram only at time instants/intervals when signal samples $x(n)$ are available. This procedure will be illustrated with example.

Example 5: Consider the signal defined in Example 3, and assume that samples from the intervals 20-28, 50-80, and 95110 are missing. Since the total number of samples is 129 we have $43 \%$ of missing samples. The estimation results obtained by using the available signal values are presented in Fig. 5. Regions with unavailable samples are presented with white color in this figure. The parameters estimated using available samples are

$$
\begin{aligned}
& \hat{f}_{m}^{(1)}=\frac{6.4}{2 \pi} \approx 1.401, \quad \hat{A}_{m}^{(1)}=49.4, \quad \hat{\theta}_{m}^{(1)}=30.8^{\circ} \\
& \hat{f}_{m}^{(2)}=\frac{6.4}{2 \pi} \approx 0.986, \quad \hat{A}_{m}^{(2)}=35.0, \quad \hat{\theta}_{m}^{(2)}=-58.3^{\circ} \\
& \hat{f}_{m}^{(3)}=\frac{6.4}{2 \pi} \approx 0.796, \quad \hat{A}_{m}^{(3)}=28.5, \quad \hat{\theta}_{m}^{(3)}=-178.7^{\circ} .
\end{aligned}
$$



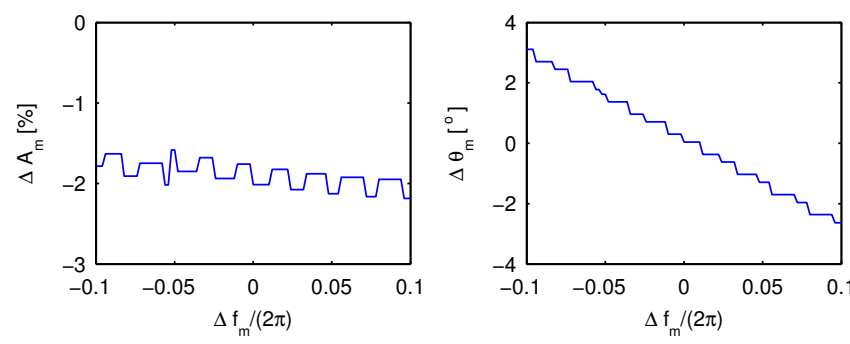

Fig. 6. Error in modulation amplitude (left) and modulation phase (right) caused by error in modulation frequency estimation.

Even with a reduced number of available signal values the presented method produced accurate estimates.

\section{E. Discretization Error Analysis}

The estimation error can be caused by many factors, for example noise, interferences, used time-frequency representation ... Here we will analyze the errors caused by parameters discretization for noise-free case only.

For modulation, the frequency estimation error is determined by a discretization step for $\alpha$, denoted by $\Delta \alpha$. Since we use $M$ equally spaced points between $f_{\min }$ and $f_{\max }$ this error can be estimated as

$$
\left|\Delta \hat{f}_{m}\right|<\frac{f_{\max }-f_{\min }}{2(M-1)}=\frac{\Delta \alpha}{4 \pi} .
$$

In the considered examples $\alpha$ is varied from 0.2 to 15 with a step 0.2 producing the discretization error in $\hat{f}_{m}$ as $\left|\Delta \hat{f}_{m}\right|<$ 0.0159 .

The modulation amplitude and phase are estimated from the IRT. The estimation error depend on the frequency discretization step in the used time-frequency representation. For a signal sampled in the time domain with $\Delta t$ and $N_{w}$ frequency points in the discrete TFR we get the estimation error for $\hat{A}_{m}$, caused by the discretization, as

$$
\left|\Delta \hat{A}_{m}\right|<\frac{1}{2 N_{w} \Delta t} .
$$

In the considered examples we have used $\Delta t=1 / 128, N_{w}=$ 55 (Example 1) producing $\left|\Delta \hat{A}_{m}\right|<1.16$. Value $N_{w}=101$ is used in other examples, producing $\left|\Delta \hat{A}_{m}\right|<0.63$. The estimation errors in our examples are slightly beyond this limits. Increase is due to instantaneous frequency bias in the spectrogram.

Phase discretization error depends on the estimated modulation amplitude. The upper limit of its absolute value is

$$
\left|\Delta \hat{\theta}_{m}\right|<\frac{180}{\pi} \frac{\left|\Delta \hat{A}_{m}\right|}{\hat{A}_{m}}
$$

resulting in: $\left|\Delta \hat{\theta}_{m}\right|<1.3^{\circ}$ for data in Example 1 and $\left|\Delta \hat{\theta}_{m}\right|<0.7^{\circ}$ for the data in Example 2 and the first estimated component in Example 3. For the second component in Example 3 we have $\left|\Delta \hat{\theta}_{m}\right|<1^{\circ}$, while $\left|\Delta \hat{\theta}_{m}\right|<1.3^{\circ}$ for the third component in this example.
Errors in $\hat{A}_{m}$ and $\hat{\theta}_{m}$ depend on error in $\hat{f}_{m}$. This dependence is presented in Fig. 6. Error in the modulation amplitude estimation has negative bias caused by the bias in the spectrogram i.e., the spectrogram maximum position is shifted from the true IF due to nonlinear modulation [12]. Here, the adaptive window could be used [13].

\section{CONCLUSION}

A method for estimation of the parameters of sinusoidally modulated signal is introduced. The proposed method is based on the inverse Radon transform and the concentration measures. It is shown that proposed method provides promising estimation and decomposition results for monocomponent and multicomponent signals. The noise and interferences influence to the estimation procedure is considered. It can be concluded that the proposed method is very robust to the noise and other interferences. We have also shown that the results obtained by the proposed method are meaningful even in cases when the analyzed signal is periodic but not sinusoidally modulated. It can be used to estimate the parameters of periodic extension of a non-periodic time-frequency patterns and partially available data as well.

\section{REFERENCES}

[1] S. Barbarossa, "Analysis of multicomponent LFM signals by a combined Wigner-Hough transform," IEEE Trans. on Signal Processing, Vol. 43, No. 6, June 1995, pp. 1511-1515

[2] S. Barbarosa and O. Lemoine, "Analysis of nonlinear FM signals by pattern recognition of their time-frequency representation," IEEE Signal Processing Letters, Vol. 3, No. 4, Apr. 1996, pp. 112-115

[3] L. Stanković, T. Thayaparan, M. Daković, and V. Popović-Bugarin, "Micro-Doppler removal in the radar imaging analysis," IEEE Trans. on Aerospace and Electronics Systems, Vol. 49, No. 2, Apr. 2013, pp. 1234- 1250

[4] L. Stanković, T. Thayaparan and I. Djurović, "Separation of Target Rigid Body and Micro-Doppler Effects in ISAR Imaging," IEEE Trans. on Aerospace and Electronics Systems, Vol. 42, No. 7, Oct. 2006, pp. 1496-1506

[5] B. Ristic and B. Boashash, "Kernel design for time-frequency signal analysis using the Radon transform," IEEE Trans. on Signal Processing, Vol. 41, No. 5, May 1993, pp. 1996-2008

[6] S. Stanković, I. Djurović, and I. Pitas, "Watermarking in the space /spatial-frequency domain using two-dimensional Radon-Wigner distribution," IEEE Trans. on Image Processing, Vol. 10, No. 4, Apr. 2001, pp. 650-658

[7] L. Stanković, "A measure of some time-frequency distributions concentration," Signal Processing, Vol.81, No.3, Mar. 2001, pp. 621-631

[8] J. C. Wood and D. T. Barry, "Linear signal synthesis using the RadonWigner distribution," IEEE Trans. on Signal Processing, Vol. 42, No. 8, Aug. 1994, pp. 2105-2111

[9] J.C. Wood and D.T. Barry, "Radon transformation of time-frequency distributions for analysis of multicomponent signals," IEEE Trans. on Signal Processing, Vol. 42, No. 11, Nov. 1994, pp.3166-3177

[10] G.T. Herman, Image Reconstruction from Projections: The Fundamentals of Computerized Tomography, Academic Press, New York, 1980

[11] L. Stanković, M. Daković and T. Thayaparan, Time-Frequency Signal Analysis With Applications, Artech House, Boston, 2013

[12] L. Stanković, M. Daković and V.N. Ivanović, "Performance of spectrogram as IF estimator,” Electronics Letters, Vol. 37, No. 12, June 2001, pp. 797-799.

[13] L. Stanković, "Performance Analysis of the Adaptive Algorithm for Bias-to-Variance Trade-off," IEEE Trans. on Signal Processing, Vol. 52, No. 5, May. 2004, pp.1228-1234 